\title{
A. y la radio escacharrada. La reducción causal en un caso de psicosis en ancianos.
}

A. and the broken radio. Causal reduction in a case of psychosis in the elderly.

\author{
Álvaro Múzquiz Jiménez a . \\ ${ }^{a}$ MIR de Psiquiatría. IPSSM José Germain. \\ Correspondencia: Álvaro Múzquiz Jiménez (alvaromuzquiz@hotmail.com) \\ Recibido: 01/11/2010; aceptado con modificaciones: 22/01/2011
}

\begin{abstract}
RESUMEN: En el presente trabajo se intenta dar cuenta de distintas aproximaciones al caso de una paciente a la que presuponemos una causa orgánica para el trastorno presentado. Para ello se utilizan las obras de Kurt Schneider y Karl Jaspers como marcos teóricos desde los que realizar tal ejercicio; intentando, finalmente, librarnos de un abordaje que sólo incluya las causas para encontrar otro que pueda hacerse cargo de la experiencia, en su integridad, de la paciente.

PALABRAS CLAVE: psicosis, alucinaciones, ancianos, causalidad.
\end{abstract}

\begin{abstract}
In this work we try to explain different approaches to the case of a patient, whose illness is assumed to be caused by an organic impairment. To that end we use Kurt Schneider's and Karl Jasper's writings as theoretical backgrounds, in an attempt to avoid an approach that only considers causes, favouring, rather, another that accounts for the patient's experience.

KEY WORDS: Psychosis, hallucinations, elderly, causality.
\end{abstract}

\section{Introducción}

En la práctica psiquiátrica nos encontramos con un panorama guiado por los actuales sistemas de clasificación diagnóstica, sistemas que agrupan signos y síntomas remitiéndolos a un diagnóstico. Este diagnóstico remite a su vez a algo que llamamos causa y que consideramos lo más profundo y lo que determina toda aquella experiencia que tenemos delante. Todo está sujeto a leyes naturales que limitan la producción humana a su facticidad, y por tanto, a puras leyes causales que, en último término, provienen del órgano donde se considera se producen las enfermedades mentales: el cerebro. Lo orgánico ya no constituye las condiciones de posibilidad para que existan determinadas experiencias, razonamientos, etc., sino que contiene en sí mismo todo en potencia. Lo demás no sería más que el producto de esa potencialidad. Frente a pacientes diagnosticados como "orgánicos" ya no cabe otra intervención que actuar sobre esa organicidad. Frente a los considerados como de causa psicológica se intervendrá de otra manera, quizá escuchándolos, pero sin abandonar el actuar sobre causas, aunque de otro tipo, en espera de que se descubran con el desarrollo de las neurociencias los mecanismos orgánicos subyacentes a lo psicológico que den paso a otra terapéutica. 
En el presente artículo presentamos el caso de una paciente a través del cual nos preguntaremos si existe otra posibilidad de abordarlo más allá de atender a la supuesta causa de sus alteraciones de la experiencia.

\section{La paciente}

A. es una mujer de 72 años que acude a la consulta del Centro de Salud Mental derivada por su Médico de Atención Primaria por posibles alucinaciones auditivas. Como tantas mujeres de su edad, A. ha ido sumando, a lo largo de los últimos años, varios tratamientos farmacológicos (simvastatina, propranolol, omeprazol, además de antiinflamatorios y analgésicos), para factores de riesgo y enfermedades (hipertensión arterial, hipercolesterolemia, artrosis, temblor). De próxima adquisición serán unos audífonos para intentar mejorar una hipoacusia bilateral que padece desde hace más de veinte años. Acude sola, sonriente, y demostrando una buena disposición a relatar lo que le ocurre. En cierto sentido, el lugar le es familiar, acudió a él en dos ocasiones durante los últimos veinticinco años.

Tercera de cinco hermanos, A. se casó y tuvo dos hijos varones que ahora tienen 49 y 50 años y que le han dado cuatro nietos. En las entrevistas siempre acaba hablando de su madre. Fue ella la figura más presente, principalmente los últimos años, ya que convivió con ella desde 1973 (fecha en que falleció su padre), hasta 1995, cuando su madre fallece repentinamente por un ACV.

Es en los años 60 cuando A. sitúa un desajuste en la forma de configurarse el mundo a su alrededor, desajuste que determinará su malestar durante muchos años. En aquella época comenzó una difícil y conflictiva historia conyugal que continúa hasta ahora. A partir de ese momento comenzó su habitual consumo de alcohol ("lo hacía para desahogarme").

Hasta la primera ocasión en que acudió al CSM llevaba años bebiendo 1,5 L de vino al día, acompañado ocasionalmente por algún vaso de brandy. Los periodos de consumo y abstinencia se alternaban por meses. Comenzó tratamiento de desintoxicación; ocho meses más tarde fue dada de alta.

Aproximadamente diez años más tarde volvió a solicitar tratamiento. Llevaba dos años bebiendo de nuevo. Su madre había fallecido tres años antes y hacía sólo unos meses que su hijo se había divorciado y ella no estaba de acuerdo con la forma en que estaba manejando esta circunstancia: volvía a necesitar "un desahogo". Tras varios meses abandonó el consumo de alcohol y fue dada de alta.

La tercera ocasión en que regresa al CSM es la que nos ocupa. Ha pasado un tiempo similar al que transcurrió entre las otras ocasiones, sus problemas se mantienen: la relación con su marido, el recuerdo de su madre, su constante estar 
NOTAS CLÍNICAS

pendiente de sus hijos. El motivo por el que acude ha cambiado. Ya no es el alcohol, que abandonó hace ya 12 años, sino unas "voces" que la vienen angustiando desde hace dos meses lo que la trae a consulta.

Aparecieron de improviso, repentinamente, una noche de finales del verano, al acostarse. Había mantenido una discusión con los vecinos por un asunto trivial de la comunidad. Al principio eran música, canciones populares que había oído de niña, voces de gente irreconocible, que aparecían en la noche cuando todo estaba en silencio y ella preparada para dormir. Creía que podrían ser los vecinos quienes generaran tanto estruendo. Pasaron los días y los sonidos aparecían con más frecuencia (durante el día), e iban adoptando otras formas y claridad: el "Cara al sol" o el rosario, que rápidamente reconoció que lo rezaba un locutor de radio ¿Qué podía hacer ella si sus vecinos, o quién sabe quién, estaban poniendo la radio con semejante contenido a ese volumen? ¿Qué podía hacer ante la angustia ocasionada? Llamó a la policía pero ellos no oyeron nada una vez allí.

El mayor de sus hijos insistía en que lo que oía no era la radio de nadie, ni música, ni sonidos que provinieran de ningún sitio o que produjera alguien. Su hijo le hizo ver que lo que oía ya lo conocía de antemano, que no oía nada novedoso, nada que no hubiera oído antes. De esta manera fue tomando conciencia, y, con la participación de algún vecino, llegó a convencerse de que aquello que oía era un producto suyo, que eran "voces que no existían", y acudió al médico. Su médico pautó tratamiento con Haloperidol, que toleró bien, y le ayudó a disminuir en parte la sintomatología y rebajar su angustia. Los audífonos terminan ese trabajo y ayudan a que las alucinaciones prácticamente desaparezcan.

\section{Primera aproximación}

Si queremos realizar un planteamiento y manejo paradigmático del terreno en que nos movemos en la práctica diaria comenzaremos por un resumen de las características principales que se pueden recoger de la paciente y orientarnos hacia un diagnóstico y tratamiento concretos.

A. es de sexo femenino y de una edad avanzada (72 años). Los antecedentes médicos que más relevancia tienen para sus síntomas actuales son la hipoacusia y el abuso de alcohol durante años. Presenta unos síntomas que se podrían identificar como alucinaciones auditivas mantenidas en el tiempo, de carácter egodistónico, para las que no presentaba "insight" aunque lentamente lo fue adquiriendo. Sus sospechas acerca de que dichos sonidos fueran provocados por los vecinos difícilmente pueden encuadrarse dentro de la esfera paranoide. Aparentemente no presenta trastorno afectivo alguno ni deterioro cognitivo objetivable. Mejora con tratamiento neuroléptico y unos audífonos. 
Con esta información ¿Cuál es el paso siguiente? ¿Qué nos exige nuestra posición como médicos o psicólogos? Antes hemos dicho que nos orientábamos hacia un diagnóstico y un tratamiento posterior desde la información recogida con la que operaríamos en adelante. Por ahora no queremos desviarnos de ese propósito, así que lo siguiente será encontrar algún diagnóstico que nos oriente.

Si revisamos parte de la literatura referente a síntomas psicóticos en ancianos (1-4), nos encontramos con un procedimiento muy similar, que no ha variado a pesar de los años transcurridos, lleguen o no los autores a las mismas conclusiones, o aborden asuntos dispares. En todos rige una guía como la aquí expuesta: delimitación de entidades diagnósticas y la utilidad de las mismas, búsqueda de síntomas específicos para esas entidades, relación de los síntomas y los diagnósticos con lesiones orgánicas, y, por último, la determinación del tratamiento más eficaz.

Comenzando por el primer punto, los diagnósticos que se manejan son: 1.Una forma de presentación atípica del Síndrome de Charles Bonnet -que remitiría generalmente a una lesión orgánica-, 2. otros trastornos orgánicos, 3. psicosis afectivas, 4.esquizofrenia o parafrenia tardías, 5. alucinaciones no patológicas.

Sin entrar en mayores disquisiciones que nos ocuparían quizá demasiado tiempo y espacio, afirmamos que la mejor etiqueta-diagnóstico es el síndrome de Charles Bonnet, una forma de alucinosis, pero con una presentación no típica (auditiva frente a la visual clásicamente descrita). Ante este cuadro, la lógica seguida nos lleva a actuar sobre aquellos componentes biológicos que suponemos alterados: el cerebro y la vía auditiva. Así utilizamos las herramientas de que disponemos: audífonos y haloperidol. Posteriormente discutiríamos acerca de la localización concreta de la supuesta lesión, de las pruebas a realizar en busca de ésta o de la exactitud o no del diagnóstico establecido y tratamientos utilizados. De esta manera se cerraría el caso.

\section{Cuestiones y alternativas}

Hasta aquí hemos seguido el modo más o menos natural en que nos desenvolvemos con un paciente de semejantes características. Es en este momento cuando surgen las preguntas: ¿es éste el proceder más correcto?, ¿nos hemos acercado así por algún motivo, por alguna clase de mirada prejuiciosa?, ¿es en este momento cuando está definido totalmente el otro, su ser, a través de un diagnóstico y su causalidad biunívocas?, ¿deberíamos continuar indagando? Podría objetarse que este tipo de cuestiones no tienen mayor relevancia, ya que nuestra tarea es "curar" y es éste el procedimiento seguido hasta ahora. Un buen procedimiento que nos lleva a diagnósticos y tratamientos eficaces. Estaríamos parcialmente de acuerdo si fuera 
NOTAS CLÍNICAS

cierto (aunque no por ello deberíamos abandonar cualquier tipo de discusión al respecto), pero en este tipo de casos ni siquiera tenemos una evidencia terapéutica tal que justifique semejante afirmación (1-4).

Para ser capaces de responder a las preguntas habrá que rastrear y replantearse el lugar desde el que nos hemos aproximado y encontrar algunos lugares de autoridad y creadores de discurso desde los que hayamos tomado -quizá sin darnos cuenta-, esta manera de actuar. Para ello utilizaremos el texto de K.Schneider "Psicopatología clínica" (5) como ejemplo de la lógica que ha guiado nuestra actuación hasta aquí y veremos que desde él se dirige toda aproximación por una forma de mirar.

\section{K.Schneider}

"Es imposible tener alguna clase de visión de la psicopatología clínica si antes no han quedado claras dos cosas. Primera: que hay, por un lado, anormalidades psíquicas que son variedades anormales del psiquismo, y que hay, por otro lado, anormalidades psíquicas que son consecuencia de enfermedades."(5)

Schneider, al comienzo de su libro, nos sitúa en las coordenadas desde las que nos deberemos mover a partir de ese momento. Este terreno es el de las causas y consecuencias. Reformulándolo: es imposible cualquier acercamiento clínico sin atender primero a la causalidad de los síntomas y cuadros clínicos.

Siguiendo este presupuesto el siguiente paso es la clasificación de dichos cuadros en uno u otro grupo y la discusión y polémica acerca de dónde situar algunos de ellos (esquizofrenia y ciclotimia). Una vez hecho esto iríamos ya al fondo, al estudio de la causalidad concreta de cada cuadro; causas psicológicas en uno u orgánicas en otro.

Schneider nos da las pautas concretas a seguir desde su llamada sistemática clínica e indica cómo se construye un diagnóstico*, y la dirección que deben tomar la psicopatología y psiquiatría en este cometido:

"La experiencia señala que construir un diagnóstico psiquiátrico a partir de hallazgos psicopatológicos presenta a menudo dificultades muy grandes (...) No se trata de sumar y combinar síntomas que pueden ser aprehendidos y mostrados objetivamente, sino de dar un dictamen sobre declaraciones hechas por el sujeto

\footnotetext{
* No olvidamos la distinción establecida entre psicopatología general y clínica (6), ni pretendemos medir a la segunda desde la primera; pero eso no impide que estudiemos lo que implican y las consecuencias en la práctica clínica de ambas disciplinas.
} 
investigado, de usar el criterio de valoración el modo de portarse o comportarse un sujeto y las impresiones recibidas por el examinador"

Siguiendo a Schneider, la dificultad con que nos topamos en nuestra tarea se distingue de la del resto de médicos por la naturaleza de los síntomas (faltos de objetividad), siguiendo a partir de ahí una ruta similar o idéntica a la de cualquier otra rama de la medicina: agrupación de esos síntomas y dictamen diagnóstico posterior. No es extraño que prosiga sugiriendo que lo somático prima sobre lo psíquico en el diagnóstico y que la psiquiatría debe encontrar cada vez más signos somáticos para hacer sus diagnósticos (así ya no existirían ni siquiera las diferencias y dificultades encontradas por él).

Decíamos que utilizábamos el texto de Schneider como ejemplo o sustento teórico-discursivo de autoridad sobre el que nos apoyábamos para proceder como lo hacíamos. Si esto es así será posible utilizarlo como un esquema en el que incluir los datos concretos que aquí nos comprometen (figura 1). Primero agrupamos los síntomas, signos y factores de riesgos psíquicos y somáticos (siguiendo la terminología dualista de Schneider): alucinaciones con buen "insight", sin aparente deterioro cognitivo, sintomatología delirante, ni afectiva mayor, hipertensión arterial, hipercolesterolemia, hipoacusia e historia de abuso de alcohol. Todos estos factores aparentemente aislados los agrupamos y miramos hacia donde nos dirijan, esto es, hacia un origen orgánico de los síntomas. Y en este punto, aparentemente todo resuelto, procedemos a un tratamiento que corrija o enderece parte de esas causas orgánicas, y, como efecto, los síntomas.

Figura 1

\section{SUMA DE SÍNTOMAS, SIGNOS, FACTORES DE RIESGO}

\section{ETIOLOGÍA}

Alucinaciones con buen "insight".

Hipoacusia.

No aparente deterioro cognitivo.

No sintomatología delirante.

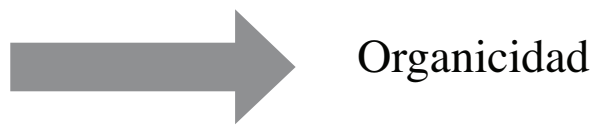

No sintomatología afectiva mayor.

HTA.

Hipercolesterolemia.

Alcohol.

El problema es que, aun suponiendo que esta relación fuera cierta, la hemos hecho guiados por un prejuicio, que, como hemos dicho antes, consiste en dirigir nuestra mirada constantemente sobre las causas y tener que clasificar lo que nos 
encontramos con el paciente en uno de los grupos propuestos. Desde este proceder establecemos una relación sobre la que no hay evidencia pero que aceptamos y operamos con ella. Se establece la causa orgánica que determinará toda la actuación siguiente sin necesidad de pruebas dirigidas en ese sentido (que aunque detectaran una alteración, tampoco en cualquier caso constituirían una evidencia incontestable), como un hecho cierto y que otorga inteligibilidad y sentido a lo que antes parecía una mezcla sin ordenar.

La realidad es que esto es lo que habría ocurrido si hubiéramos seguido completamente a Schneider; pero no hemos sido tan rigurosos. Schneider nos previene de un posible error en el que podríamos caer, y este es, a saber, que no deben realizarse diagnósticos clínicos como prejuicios y posteriormente valorar los síntomas en función de aquello que previamente hemos diagnosticado. En nuestro caso parece que hayamos podido pasar esto por alto y hemos actuado de la siguiente manera (figura 2): la edad avanzada (72 años), nos ha hecho suponer una causa orgánica desde la que hemos reinterpretado toda la esfera alucinatoria, afectiva, etc. de la paciente.

\section{SUMA DE SÍNTOMAS, SIGNOS, FACTORES DE RIESGO}

\section{ETIOLOGÍA}

Alucinaciones con buen "insight".

Hipoacusia.

No aparente deterioro cognitivo.

No sintomatología delirante.

No sintomatología afectiva mayor.

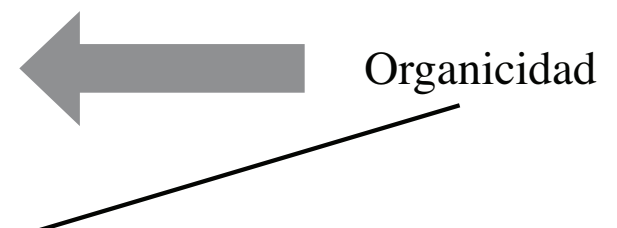

EDAD

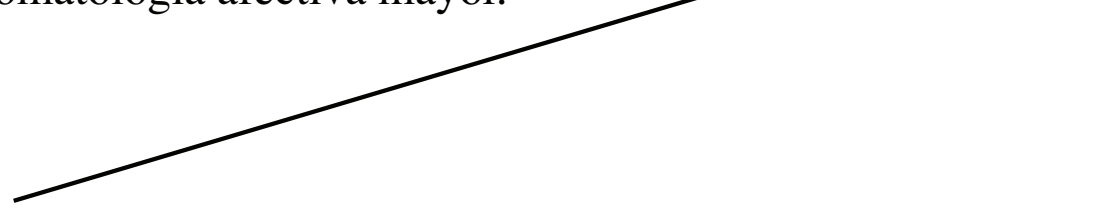

Resumiendo, hemos vaciado el discurso totalmente de su contenido, o, en el mejor de los casos, lo hemos remitido a una causalidad desde la que se torna inteligible: posibles lesiones orgánicas, causalidades del contenido como elementos de su biografía, la morfología o estructura de la vía auditiva (teorías entópicas). No hemos hecho ninguna aproximación a la experiencia que posee la paciente sobre lo que le está ocurriendo, sobre el significado que poseen para ella los síntomas, sobre su mundo. Los límites que nos propone el remitirnos constantemente a las causas, trae consigo el indagar únicamente sobre el sentido de la experiencia subjetiva en 
aquellos casos en que supongamos una causa de índole psicológica. Desde esta perspectiva, suponiendo una causa orgánica, la subjetividad del paciente pierde todo su valor.

\section{La posibilidad de escuchar "lo orgánico"}

Hasta este momento uno se mueve en una confusión entre condiciones de posibilidad (base biológica, y más concretamente un cerebro, para que exista una subjetividad) y relación causal (que los síntomas psicopatológicos estén causados por una alteración de esa base biológica), como si esta relación estuviera establecida a priori de manera necesaria. Dando primacía a estas condiciones de posibilidad, creyendo que sólo sobre ellas se puede establecer un auténtico conocimiento, enmudecemos el único hecho que realmente se nos presenta: la experiencia del otro. Pero, ¿existe alguna necesidad de estudiar esto que se nos aparece, la experiencia de la paciente? ¿Podemos encontrar algún camino cierto y un auténtico conocimiento sobre ese terreno?

En cuanto a la primera de las cuestiones encontramos distintos argumentos que han demostrado las dificultades o incluso la incapacidad para poder reducir la subjetividad (7-9). En este sentido, nuestro estudio sobre las causas orgánicas de la experiencia de la paciente se nos muestra ahora como un salto deficiente, basado en una tradición médica que considera, sin más, la enfermedad una manifestación de alteraciones orgánicas (11-13), y, que desde nuestra tendencia a utilizar lo familiar (8), tendemos a usar como patrón. En cualquier caso, se nos pide que tomemos en serio el acto intencional del sujeto en cuestión, independientemente de su base fáctico-biológica (10). Es decir, el origen orgánico o no de lo consciente de nuestra paciente no quita ni pone nada a su subjetividad.

Así para dar respuesta a la segunda de las cuestiones, si no queremos dar ese salto sino estudiar esa realidad, esa experiencia, con que decíamos que nos topábamos antes, tendremos que encontrar otros recursos para hacerlo. Distintas propuestas se han planteado en este sentido: desde una recuperación del lugar central de la psicopatología y de la vida en la psiquiatría $(12,14)$, hasta modelos hermenéuticos concretos en el abordaje de las demencias, paradigma de lo orgánico (15). Nosotros nos inclinamos por utilizar la propuesta de Karl Jaspers en su "Psicopatología general"(16). Lo hacemos por varios motivos. Jaspers es un antecesor directo de Kurt Schneider. Es a través de este último desde el que se suele interpretar al anterior y a ambos se les inscribe en lo que viene a llamarse una "psicopatología objetivante de la escuela de Heidelberg" (12). Es una de las figuras más influyentes de la historia de la psicopatología y una de las que se han enfrentado al problema de la 
NOTAS CLÍNICAS

totalidad $(12,13)$. Por último, intenta establecer una investigación firme y encontrar un conocimiento sólido del hecho psicopatológico como tal, como elemento central de la práctica psiquiátrica.

Jaspers comienza la segunda parte de su tratado ("Die verständliche Zussamenhänge des Seelelebens"), tras haber analizado en la primera las manifestaciones psicopatológicas, preguntándose acerca del origen de aquellos fenómenos que han ocupado todas las páginas anteriores:

"En la primera parte conocimos los elementos singulares que podíamos representarnos intuitivamente, ya sea como datos subjetivos de la vida psíquica realmente vividos (fenomenología) o que podíamos captar objetivamente como rendimientos palpables, como síntomas somáticos de lo psíquico, como hechos típicos significativos en la expresión, el mundo y la obra (psicopatología objetiva). En el primer plano de nuestro interés estaba la descripción de los hechos típicos. Pero en todas partes comenzaron los problemas: ¿de dónde procede la manifestación?, ¿con qué otras está en relación?"(16)

Esta pregunta sobre el origen es aparentemente una pregunta acerca de la causalidad de los fenómenos y es, a través de la incursión en ese terreno, como llega a conclusiones distintas a las que llega su discípulo. Esto es, a dos conceptos y modos de estudio diferenciados: comprender (Verstehen) y explicar (Erklären). Es el primero de ellos (comprender) el que constituiría la forma de estudio e investigación específica de la psicopatología, el que preserva el propio objeto de estudio:

"1. Por la penetración en lo psíquico comprendemos genéticamente cómo surge lo psíquico de lo psíquico. 2. Por la anudación objetiva de hechos típicos diversos en regularidades, con base en las experiencias reiteradas, explicamos causalmente." (...) "nunca podemos, como en la física y la química, establecer ecuaciones causales. Esto supondría una completa cuantificación de los procesos examinados, que en lo psíquico, que según su esencia permanece siempre cualitativo, no es posible nunca, en principio, sin que el verdadero objeto de la investigación, es decir el objeto psíquico, se pierda” (...) "Mientras en las ciencias naturales sólo pueden ser halladas relaciones causales, en psicología, el conocer encuentra su satisfacción en la captación de una especie muy distinta de relaciones."

Inscrito en una tradición psiquiátrica con pretensiones de objetividad trata, de todos modos, de librarse de un positivismo ingenuo con este método y construir un conocimiento auténtico y evidente:

"La evidencia de la comprensión genética es algo último." (...) "Tal evidencia es adquirida con motivo de la experiencia frente a las personalidades humanas, pero no por la experiencia que se repite, inductivamente probada" (...) "Relaciones genéticamente comprensibles, en cambio, son relaciones de tipo ideal, son evidentes en sí (no adquiridas inductivamente), no conducen a teorías." 
Este proceder podemos entenderlo bien como la formulación de juicios sintéticos a priori (17), o como el descubrimiento y descripción de partes no-independientes $(10,18)$, del psiquismo "alterado". La causalidad por la que parecía interrogarse ya no se presenta como tal y lo que en un primer momento creíamos era la única respuesta a la pregunta (“¿de donde procede la manifestación?”), se abandona, retomándola solamente en caso de que se agote la vía de la comprensión: "todo límite de la comprensión es un nuevo estímulo para la interrogación causal”, pero sin olvidar nunca la primacía de esta vía sobre la de las causas*.

Por lo tanto, si queremos tomar en serio el discurso, la experiencia, la conciencia de nuestra paciente y no alterar ni perderlo se nos torna claro que el procedimiento que seguimos al principio no era el más adecuado. Parece que más bien lo que situábamos como comienzo debe ser lo último, y que primero deberíamos realizar una labor totalmente distinta. En palabras de Jaspers: comprender.

\section{Inicio de una comprensión de A.}

Lo primero que encontramos siguiendo estas nuevas coordenadas es un relato biográfico y su angustia y malestar durante años en relación con el mismo: los años en que sitúa el inicio de las dificultades conyugales, cómo entonces ella comenzó a trabajar, se hizo cargo de sus hijos, las dificultades que continuaron y cómo su madre fue la persona que le sirvió de apoyo durante todos los años más complicados. $\mathrm{El}$ alcohol se introduce como uno de los elementos en sus intentos de manejar la situación, de afrontar la angustia y mitigarla de alguna manera.

Avanzando y ocupándonos de lo que la trajo como motivo de consulta nos situamos en el síntoma actual: las alucinaciones. Ella las sitúa dentro del relato biográfico. Angustiada y triste desde hacía muchos años, estas sensaciones aumentaron su intensidad en el momento en que su hijo se separó de su mujer: no le gustó la actitud de su hijo. Dice que desde ese momento rumia los problemas, es "un darle vueltas a las cosas" y de tanta angustia, de "tanto darle vueltas" y empeorar con la discusión con los vecinos ("un encontronazo"), los problemas salieron fuera como "respuesta" o "parte" de "la depresión", como hacía doce años cuando "lloraba sin parar y le parecía que su hijo aparecería en cualquier momento", y se presentaron en forma de alucinación.

\footnotetext{
* “Contra la generalización absoluta del conocimiento causal." p.383 de la edición alemana.
} 
NOTAS CLÍNICAS

La repercusión inicial que supusieron estas "voces y canciones" fue el temor que le provocaron: creía que podría estar sufriendo un ACV como su madre o que sus vecinos "querían molestarla". Más tarde, al darse cuenta de que esto no era así, se tranquilizó y empezó a intuir qué era todo aquello, de dónde provenían esos sonidos y cuál era la referencia del contenido de los mismos: su madre.

Como señalamos antes, su madre fue la figura principal durante la mayor parte de su vida, y, como centro, así se presentaba también en "las voces". El Rosario era "lo que le rezaba a mi madre de niña para que se tranquilizara", el "Cara al sol" era una parte de "las historias de mi madre" y las canciones populares "las que cantaba de niña con mi madre". Es ahora, cuando de nuevo se presenta la angustia, cuando su madre vuelve a aparecer, de un modo extraño, pero que le recuerda lo familiar.

Empieza a mostrarse la naturaleza comprensible de su experiencia. Si continuamos podría seguir contando cómo incorpora estos síntomas a su vida, cómo los maneja, cómo podemos ayudarla en esa tarea reconstruyendo juntos lo ocurrido y lo que queda por ocurrir. Sólo es en el límite, al final del camino, cuando se da el salto a la causa; al final, y sin olvidar el recorrido.

\section{BIBLIOGRAFÍA:}

(1) Beck J.; Harris J. Visual hallucinosis in non-delusional elderly. Int J Geriatr Psychiatry 1994;

(2) Leuchter A.; Spar J. The late-onset psychoses, clinical and diagnostic features. J Nerv Ment Dis $1985 ; 173$ : 488-94.

(3) Soares JC, Gershon S. Therapeutic targets in late-life psychoses: review of concepts and critical issues. Schizophr Res 1997; 27: 227-39.

(4) Broadway J, Minzer J. The many faces of psychosis in the elderly. Curr Opin Psychatry 2007; 20: 551-8.

(5) Schneider K. Psicopatología clínica. Madrid Fundación Archivos de Neurobiología. 1997

(6) Rossi Monti M Stanghellini G. Psychopathology: an edgeless razor? Compr Psychiatry 1996; 37: 196-204.

(7) Zahavi D. Subjectivity and selfhood: investigating the first-person perspective. Cambridge, Massachusetts, London: Massachusetts Institute of Technology, 2005

(8) Nagel T. What is it like to be a bat? Philosophical Review 1974; 83: 435-50.

(9) Fuchs T. Das Gehirn- ein Beziehungsorgan. 2a Ed. Stuttgart: Kohlhammer, 2009.

(10) Husserl E. Investigaciones Lógicas. Madrid: Alianza, 1985.

(11) López Piñero JM; Luz Terrada M. Introducción a la medicina. Barcelona: Crítica, 2000.

(12) Ramos P, Rejón C. El esquema de lo concreto. Madrid: Triacastela, 2002.

(13) Baca E. Teoría del síntoma mental. Madrid: Triacastela, 2007.

(14) Ramos P. Vida, psiquiatría y biopolítica. Un asunto psicopatológico. Frenia, 2009; 9: 7-32.

(15) Widdershoven GAM, Widdershoven Heerding I. Understanding dementia: a hermeneutic 
perspective. En: Fulford B, Morris K, Sadler J, Stanghellini G. Nature and narrative. Oxford: Oxford University Press, 2003.

(16) Jaspers K. Allgemeine Psychopathologie. Berlin-Heidelberg-New York: Springer, 1973. (Traducción al castellano: Psicopatología General. México: Fondo de Cultura Económica, 1993).

(17) Kant I. Crítica de la razón pura. Madrid: Alfaguara, 1978.

(18) Valor Yébenes JA. Objetivismo absoluto: una crítica empirista a la filosofía trascendental. Revista de filosofía 2002; 27: 191-221. 